\title{
Study on Cutting Mechanism of Monocrystalline Silicon Using Molecular Dynamics on Nanometric Scale
}

\author{
Li-Qiu SHI ${ }^{1, a}$, Jia-Xin XIE ${ }^{2, b}$, Ming-Dong XI ${ }^{3}$, Xiao-Hai $\mathrm{LI}^{4}$, Feng $\mathrm{YU}^{5, \mathrm{c},{ }^{*}}$ \\ ${ }^{1,2,4}$ Mechanical engineering college, Jiamusi University, China \\ ${ }^{3,5}$ Educational administration, Jiamusi University, China

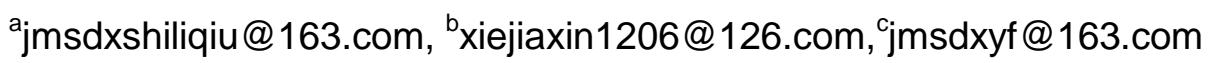 \\ ${ }^{*}$ Corresponding author
}

Keywords: Molecular Dynamics, Modeling, Mechanism of Nanometric Machining.

\begin{abstract}
Using molecular dynamics simulation method study the mechanism of nanometric cutting of monocrytalline silicon. We used LAMMPS software programming to establish model.Through VMD video software to display the image of cutting process and combining with the change of potential and temperature, we understand the machining mechanism of nanometric cutting of single crystal silicon is the deformation of amorphous phase change produced rather than plastic deformation. We also found, among cutting parameters, cutting depth has a great impact on surface quality of workpiece.
\end{abstract}

\section{Introduction}

In recent years, ultra precision machining of hard and brittle materials jumped to become the new hotspot of study. In the ultra precision machining of nanoscale, machining precision can reach nanometer level, the remove method of the workpieces have become dispersion of atoms or atomic layer, it is not a continuous solid any more. If you continue to use the traditional cutting theory to analyze this process, it must be unreasonable[1]. At the same time, since measurement, observation and control is difficult in the processing, it makes the study have some inevitable difficulties. Therefore, people put forward by means of molecular dynamics simulation to overcome these difficulties, to study and analyse the mechanism of nano fabrication from the atomic level. Its purpose is "reproduce" the nature of the real process on a computer, including the process has actually occurred and the experimental conditions are not permitted.Moreover, molecular dynamics simulation is particularly suitable for the realization of those who don't comply with laboratory experimental quantitative, but it can explain or prove some "thought experiment" of qualitative conclusions[2].

\section{The Establishment of Simulation Model}

In molecular dynamics simulation, first of all, we should model for simulation system. Reasonable setting of model will contribute to the subsequent calculation. In this paper, a set of simulation parameters display on table:

Tab. 1 Simulation parameters of monocrystalline silicon nano machining

\begin{tabular}{lcc}
\hline \hline Material & Workpiece:monocrystalline silicon & Tool:diamond \\
The Shape Of Tip [A] & Hemisphere & $\mathrm{r}=12$ \\
Potential Function & Tersoff & \\
Ensemble & NVE \\
Time Step [ps] & 0.001 \\
Speed Of Cutting [m/s] & $100,200,400$ \\
Depth Of Cutting [A] & $1,2,4$ \\
The Initial Temperature [K] & 300 \\
\hline \hline
\end{tabular}


Molecular dynamics simulation model of single crystal silicon nano processing as shown in Figure.1.

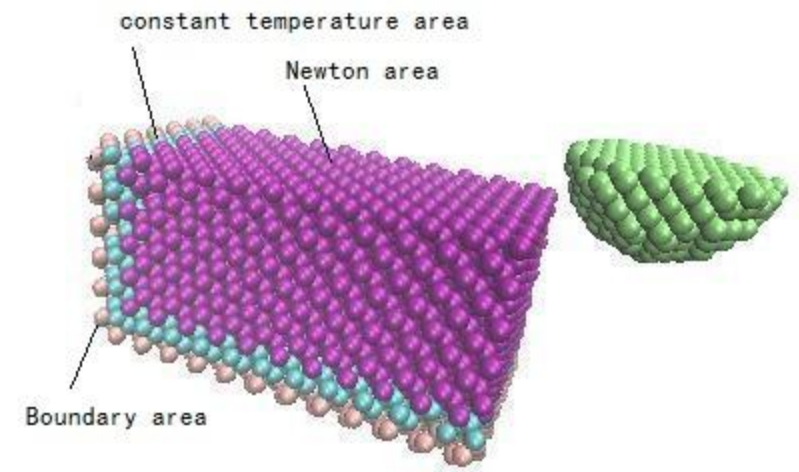

Fig.1 Molecular dynamics simulation model of monocrystalline silicon nano machining

\section{Analysis of Simulation Results}

\section{The Material Removal Mechanism and the Forming Mechanism of Machined Surface}

This paper selects the instantaneous image of cutting process, as shown in figure 2.

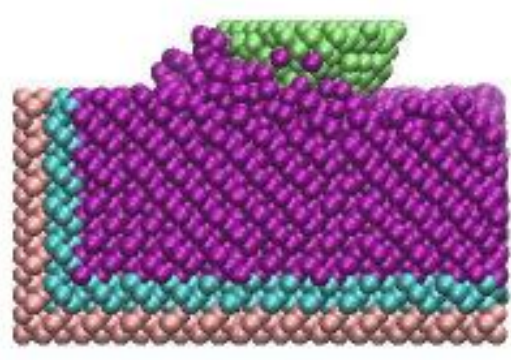

a) Side view

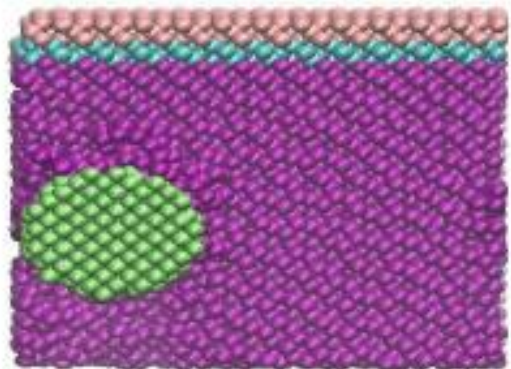

b) Vertical view

Fig.2 Instantaneous images of monocrystalline silicon cutting process

We can get the material removal mechanism and the forming mechanism of machined surface through the analysis of potential energy and temperature change in machining process, which can be interpreted as: In the cutting process, with the movement of tool, repulsive force makes the silicon lattice occurred shear extrusion deformation. Along with the repulsive force between atoms is more and more big, so the lattice deformation energy stored in the lattice will also largen, when the energy exceeds a certain value but do not form dislocation, fracture of silicon atom bond, the original structure of the lattice is broken and show amorphous. These atoms that their bonds was broken in perpendicular to the cutting direction is divided into two parts, atoms of the upper part move onward and upward and form chip, the remaining part move backward and downward and form the machined surface. As described above, the atoms whose bonds are broken moving in two directions and repeating the process of replacement is the material removal and machined surface formation process. The potential energy curve display that the process of potential energy gradually becoming large only has some small fluctuations, which indirectly indicates that nano cutting process of silicon has no dislocation. Therefore, the nano machining mechanism of silicon is deformation of amorphous phase transform generates.

\section{Analysis of Temperature and Potential Energy}

In the nano cutting process, the source of cutting temperature, in addition to transforming from deformation and friction in the cutting process, is from the conversion of kinetic energy and heat energy of silicon atoms. As shown in Figure 3, the temperature curve has fluctuations, this is because the friction of tool and the workpiece increases the kinetic energy of atoms, and atomic 
bond fracture releases energy, temperature of system rises. Along with the cutting, the fracture atomic bond combined with others to form new covalent bond, absorbing energy sent temperature down.

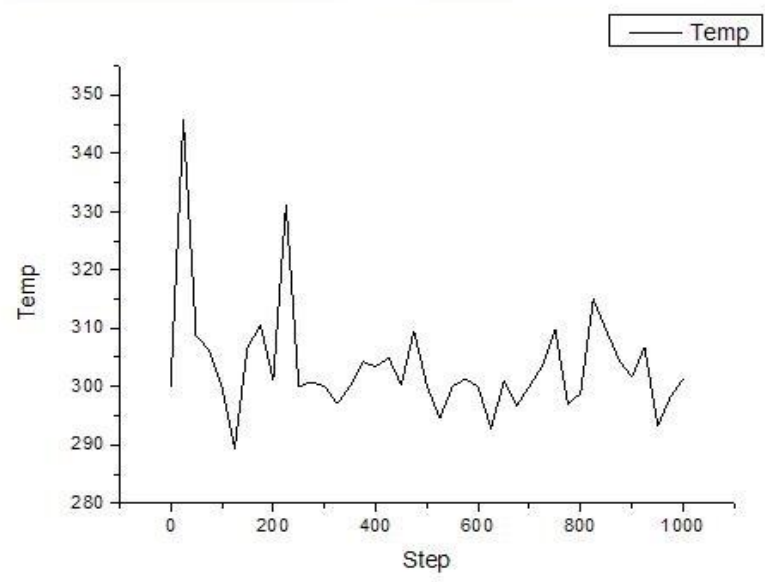

Fig.3 Temperature change curve in the cutting process

From Figure 4 curve can be seen, with the cutting, the potential energy between atoms increased, the amplitude slowed gradually with fluctuations. Because when the atoms suffering shear stress will make the atoms bond broken, and at the same time by extrusion stress, atomic lattice of the front of edge occur deformation with the deformation energy stored in the lattice, and it will continue to accumulate, thereby the potential between atoms increase. However, lattice reconstruction and the atomic elastic recovery will reduce the growth trend. And the deformation and reconstruction of lattice, forming and expansion of amorphous atoms will cause fluctuation.

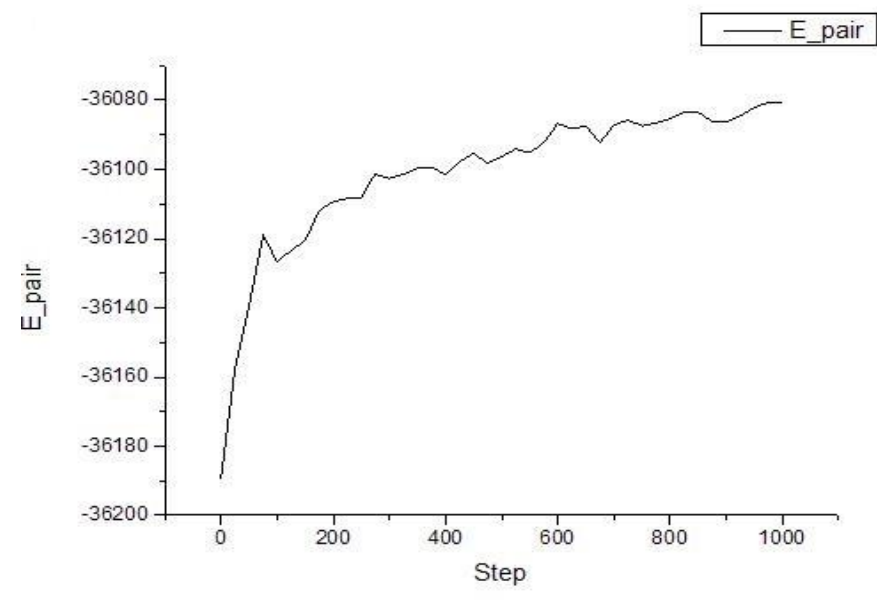

Fig.4 Potential energy curve in the cutting process

\section{The Effect of Cutting Depth on Cutting Process}

In this paper, the simulations are carried out for different cutting depth and cutting velocity, the influence of cutting speed on workpiece surface quality is not obvious. Therefore, this paper only introduced the effect of cutting depth here. In order to study the influence of different cutting depth, without tool and cutting speed changing, we choose three kinds of cutting depths to simulate and calculate, then take instantaneous images at the same cutting distance, as shown in Figure 5.

As can be seen from the picture, the increasing of cutting depth lead to accumulation of amorphous atoms in the front of tool, changing thick, and the situation of deformation around tool tip atom increase, damage layer become deeper. It is the surface becoming rough from the macro perspective. 


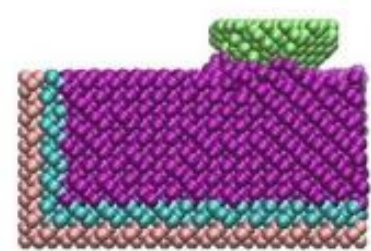

a) $1 \mathrm{~A}$

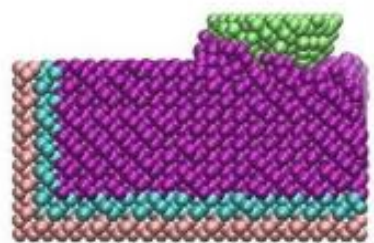

b) $2 \mathrm{~A}$

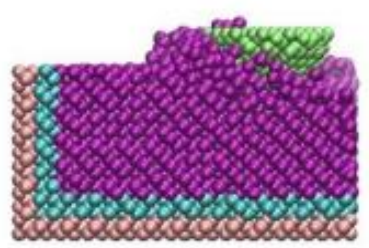

c) $4 \mathrm{~A}$

Fig.5 The instantaneous images of different cutting depth at the same cutting distance

\section{Conclusion}

(1)Using the molecular dynamics simulation, we researched and analysed the material removal mechanism and the machined surface formation mechanism of the monocrystal silicon nanometric Cutting .Under the action of the cutting edge, the silicon atomic bond broke in front of tip. And these atoms whose bond broken along the vertical direction is divided into two parts, one part move upward to be chip, the other part move down to form machined surface. Furthermore, the processing mechanism of nanometric cutting of single crystal silicon is amorphous phase transformation to produce deformation instead of plastic deformation.

(2)From the above simulation, it indicates that the influence of different cutting parameters on machining process are not the same. The influence of cutting depth on the quality of the workpiece surface is larger, with the increasing of cutting depth, the surface roughness of machined surface will increase.

\section{Acknowledgement}

This work was financially supported by the National Natural Science Foundation of China (No.51105174), the National Natural Science Foundation of China (No.51375208) and the New Century Excellent Talents to Support Plan in Heilongjiang Province Colleges and Universities (No. 1254 - NCET - 022).

\section{References}

[1] Jun Tian, YingChun Liang ect:The study of nanometric grinding process based on Molecular dynamics simulation[J]. Jiangsu Machinery Manufacturing And Automation,2001(4):139-145.

[2] YuHua Wen,RuZeng Zhu:Main technology of molecular dynamics simulation[J].Mechanical Progress,2003, 33 (1): 65-73.

[3] Yufei Gao, Peiqi Ge, Zhijian Hou.Study progress on Molecular Dynamics simulation of ultra precision machining of silicon[J]. Tool Technology, 2006, 4 (9):3- 6.

[4] YuLan Tang, ect. Study of monocrystal silicon nanometric cutting mechanism based on Molecular Dynamics[J].Microfabrication Technology, 2003 (2): 76-80.

[5] YunPeng Kou.Study on molecular dynamics simulation of monocrystal silicon nanometric cutting process[D].2010:9-10.

[6] ZhengLong Chen, WeiRen Xu, LiDa Tang. The practice and theory of molecular simulation[M]. Beijing: Chemical Industry Press,2007:67-68.

[7] Landman U,Luedtke W D,Burnham N:Atomistic mechanisms and dynamics of adhesion,nano indentation, and fracture. Science,1990,248(4954): 454-461.

[8] Komanduri R, Chandrasekaran M, Raff L M. MD simulation of nanometric cutting of single crystal aluminum-effect of crystal orientation and direction of cutting[J].Wear, 2000, 242: 60-88. 